\title{
Nanoindentation and the low velocity impact response of biofibre, biopolymer and its biocomposite derived from sugar palm tree
}

\begin{abstract}
Biofibres have gained a considerable attention due to benefits such as environmentally friendly, renewability, biodegradability, low weight and cost. Although many researches have been done on sugar palm fibres (SPF), there are few studies on characterizing their micromechanical properties by using nanoindentation. The hardness and elastic modulus of isolated SPF is $11.3 \pm 0.545 \mathrm{MPa}$ and $149 \pm 4.96 \mathrm{MPa}$ respectively. The hardness properties were found to be comparable with the other established fibres i.e. bamboo $(12.9 \pm 0.378 \mathrm{MPa})$, jute $(11.2 \pm 0.47$ $\mathrm{MPa})$ and hemp (7.2 $\pm 0.303 \mathrm{MPa})$. Then, biopolymer derived from sugar palm starch (SPS) and sugar palm fibre reinforced sugar palm starch (SPF/SPS) biocomposite were successfully developed by using hot press with the presence of glycerol as plasticizer. These environmentally friendly materials were tested for their low velocity impact response. The result shows that SPF/SPS exhibited the best impact properties compared to SPS. The impact load of composites increases with increasing impact energy for both SPS and SPF/SPS specimens.
\end{abstract}

Keyword: Biofibre; Nanoindentation; Biopolymer; Biocomposites; Low velocity impact; Starch 